\title{
Fractal Generation by 7-Point Binary Approximating Subdivision Scheme
}

\author{
Mehwish Bari $^{1 *}$, Ghulam Mustafa ${ }^{2}$, Abid Hussain ${ }^{1}$ \\ ${ }^{1}$ National College of Business Administration and Economics, Sub-Campus, Bahawalpur, Punjab, Pakistan. \\ 2 The Islamia University of Bahawalpur, Pakistan.
}

* Corresponding author. Tel.: +923027802539; email: mehwishbari@yahoo.com

Manuscript submitted October 18, 2017; accepted February 13, 2018.

doi: 10.17706/ijapm.2018.8.4.105-111

\begin{abstract}
In this paper, fractal properties of 7-point binary approximating subdivision scheme are presented. It is shown by the graphical presentation that the 7-point binary approximating scheme is helpful for fitting 2-dimensional data and has elegant well designed properties. Ranges of parameter are also defined to obtain fractals which lie inside and outside of the convex hull. Due to the parametric range, we can easily handle the limit curve according to our own choice. Since the given scheme is approximating, so we can get better smoothness as compare to interpolating scheme. Some numerical examples have been presented to fit the data points. It has been observed that 7-point scheme is quite suitable for fitting data points and a good selection for construction of fractals for the modeling purpose of decoration pieces and fabric designing etc. Nowadays one major approach of fractal is the generation of fractal antennas which are very helpful in the cell phone companies.
\end{abstract}

Key words: Approximation, data fitting, fractal, subdivision.

\section{Introduction}

Nowadays, a field of mathematics, named as Computer Aided Geometric Design (CAGD) is growing rapidly. Its vast applications in computer aided images, industry, surgical equipments and robotics. Different approaches can be use for the designing of curves/surfaces in which one is the subdivision approach.

Subdivision schemes can be divided into approximating and interpolating subdivision schemes. At every refinement level, if new points are located at the existing control polygon and initial points also remain in the subsequent sequences of limiting curve, the scheme is called interpolating otherwise approximating.

The initial efforts on subdivision scheme was by Rham [1], he worked on recursively corner cutting piecewise linear approximation techniques to attain a $\mathbf{C}^{\mathbf{0}}$-continuous limiting curve. In 1987, Dyn et al. [2] proposed 4-point interpolating scheme instead of approximating scheme. Chaikin [3] proposed corner cutting subdivision approach for curve design. In 1986, Dubuc [4] proposed a new linear interpolation method which produces twice differentiable functions. Boor [5] discovered that corner cuttin technique by Chaikin's algorithm [3] generates continuous curves. In this scheme new methods are need to check the continuity and differentiability. Weissman [6] introduced 6-point binary interpolatory subdivision scheme in (1990). Romani [7] proposed different families of approximating schemes that produce piecewise exponential polynomial. A little focus has been given to the fractal property of subdivision schemes as compare to the smoothness. Fractal properties of some well known subdivision schemes have not been explore yet. A brief survey is given below. 
Zheng et al. [8] analyze the fractal behavior of binary 4-point and ternary 3-point interpolating schemes. Wang et al. [9] worked on the fractal generation by generalized Chaikin scheme. Siddiqi et al. [10] worked on the fractal behavior of ternary 5-point interpolating subdivision scheme. Mustafa et al. [11] designed different images by fractal approach of different subdivision scheme.

The paper arrangement is given as: Section 2 presenting the 7-point binary approximating subdivision scheme, also discuss the fractal generation by the scheme. Numerical examples showing the fractal behavior of the proposed work in Section 3. Section 4 contains conclusion. Finally acknowledgement is given.

\section{7-Point Binary Approximating Scheme}

First we present the 7-point binary approximating subdivision scheme as follows

$$
\begin{aligned}
f_{2 i}^{k+1}= & \left(\frac{63}{8192}+\mu\right) f_{i-3}^{k}-\left(\frac{495}{8192}+6 \mu\right) f_{i-2}^{k}+\left(\frac{1155}{4096}+15 \mu\right) f_{i-1}^{k}+\left(\frac{3465}{4096}-20 \mu\right) f_{i}^{k}-\left(\frac{693}{8192}-15 \mu\right) f_{i+1}^{k}+ \\
& \left(\frac{77}{8192}-6 \mu\right) f_{i+2}^{k}+\mu f_{i+3}^{k}, \\
f_{2 i+1}^{k+1}= & \mu f_{i-3}^{k}+\left(\frac{77}{8192}-6 \mu\right) f_{i-2}^{k}-\left(\frac{693}{8192}-15 \mu\right) f_{i-1}^{k}+\left(\frac{3465}{4096}-20 \mu\right) f_{i}^{k}+\left(\frac{1155}{4096}+15 \mu\right) f_{i+1}^{k}- \\
& \left(\frac{495}{8192}+6 \mu\right) f_{i+2}^{k}+\left(\frac{63}{8192}+\mu\right) f_{i+3}^{k},
\end{aligned}
$$

\subsection{Continuity of 7-Point Binary Approximating Scheme}

First we will calculate the continuity of 7-point binary approximating scheme. Since the mask of the scheme is

$$
\begin{aligned}
a= & \left(\mu, \frac{63}{8192}+\mu, \frac{77}{8192}-6 \mu,-\frac{495}{8192}-6 \mu,-\frac{693}{8192}+15 \mu, \frac{1155}{4096}+15 \mu, \frac{3465}{4096}-20 \mu,\right. \\
& \left.\frac{3465}{4096}-20 \mu, \frac{1155}{4096}+15 \mu,-\frac{495}{8192}-6 \mu, \frac{77}{8192}-6 \mu, \frac{63}{8192}+\mu, \mu\right),
\end{aligned}
$$

after first divided difference of (2)

$$
\begin{aligned}
a_{1}= & \left(\mu, \frac{63}{8192}, \frac{14}{8192}-6 \mu,-\frac{509}{8192},-\frac{184}{8192}+15 \mu, \frac{2494}{8192}, \frac{4436}{8192}-20 \mu, \frac{2494}{8192},\right. \\
& \left.-\frac{184}{8192}+15 \mu,-\frac{509}{8192}, \frac{14}{8192}-6 \mu, \frac{63}{8192}, \mu, 0\right),
\end{aligned}
$$

similarly by taking sixth divided difference of (1), we get

$$
a_{6}=\left(\mu, \frac{63}{8192}-6 \mu,-\frac{364}{8192}+15 \mu, \frac{730}{8192}+15 \mu, \frac{63}{8192}-6 \mu, \mu\right), \quad \text { the parametric } \text { range for }
$$

$C^{6}$-Continuity of the scheme (1), we have $\left(-\frac{6480}{524288}<\mu<\frac{9904}{524288}\right)$.

\subsection{Fractal Approach of 7-Point Binary Approximating Scheme}

To generate the fractal behavior of 7-point binary approximating scheme, we have to calculate some basic results

First we substitute $i=-3,-2$ in (1) and by putting $i=-1$ in (1), we get 


$$
\begin{aligned}
f_{-2}^{k+1}= & \left(\frac{63}{8192}+\mu\right) f_{-4}^{k}-\left(\frac{495}{8192}+6 \mu\right) f_{-3}^{k}+\left(\frac{1155}{4096}+15 \mu\right) f_{-2}^{k}+\left(\frac{3465}{4096}-20 \mu\right) f_{-1}^{k}-\left(\frac{693}{8192}-\right. \\
& 15 \mu) f_{0}^{k}+\left(\frac{77}{8192}-6 \mu\right) f_{1}^{k}+\mu f_{2}^{k}, \\
f_{-1}^{k+1}= & \mu f_{-4}^{k}+\left(\frac{77}{8192}-6 \mu\right) f_{-3}^{k}-\left(\frac{693}{8192}-15 \mu\right) f_{-2}^{k}+\left(\frac{3465}{4096}-20 \mu\right) f_{-1}^{k}+\left(\frac{1155}{4096}+15 \mu\right) f_{0}^{k}- \\
& \left(\frac{495}{8192}+6 \mu\right) f_{1}^{k}+\left(\frac{63}{8192}+\mu\right) f_{2}^{k},
\end{aligned}
$$

by subtracting (5) from (4)

$$
\begin{aligned}
f_{-2}^{k+1}-f_{-1}^{k+1}= & \left(\frac{63}{8192}\right) f_{-4}^{k}-\left(\frac{572}{8192}\right) f_{-3}^{k}+\left(\frac{1155}{4096}+15 \mu\right) f_{-2}^{k}-\left(\frac{1155}{4096}+15 \mu\right) f_{0}^{k}+ \\
& \left(\frac{572}{8192}\right) f_{1}^{k}-\left(\frac{63}{8192}\right) f_{2}^{k},
\end{aligned}
$$

Similarly

$$
\begin{aligned}
f_{-1}^{k+1}-f_{0}^{k+1}= & \mu f_{-4}^{k}+\left(\frac{14}{8192}-7 \mu\right) f_{-3}^{k}-\left(\frac{693}{8192}+21 \mu\right) f_{-2}^{k}+\left(\frac{2310}{4096}-35 \mu\right) f_{-1}^{k}- \\
& \left(\frac{2310}{4096}+35 \mu\right) f_{0}^{k}+\left(\frac{198}{8192}-21 \mu\right) f_{1}^{k}+\left(\frac{-14}{8192}+7 \mu\right) f_{2}^{k}+\mu f_{3}^{k},
\end{aligned}
$$

Further

$$
f_{0}^{k+1}-f_{1}^{k+1}=\frac{63}{8192} f_{-3}^{k}-\frac{572}{8192} f_{-2}^{k}+\frac{1848}{4096} f_{-1}^{k}-\frac{1848}{8192} f_{1}^{k}+\left(\frac{572}{8192}\right) f_{2}^{k}-\left(\frac{63}{8192}\right) f_{3}^{k},
$$

Furthermore

$$
\begin{aligned}
f_{1}^{k+1}-f_{2}^{k+1}= & \mu f_{-3}^{k}+\left(\frac{14}{8192}-7 \mu\right) f_{-2}^{k}+\left(\frac{198}{8192}+21 \mu\right) f_{-1}^{k}+\left(\frac{2310}{4096}-35 \mu\right) f_{0}^{k}+ \\
& \left(\frac{-2310}{4096}+35 \mu\right) f_{1}^{k}+\left(\frac{198}{8192}-21 \mu\right) f_{2}^{k}+\left(\frac{-14}{8192}+7 \mu\right) f_{3}^{k}-\mu f_{4}^{k},
\end{aligned}
$$

Rearranging the above, we get

$$
\begin{aligned}
f_{1}^{k+1}-f_{2}^{k+1}= & \left(\frac{63}{8192}\right)\left(f_{-1}^{k-1}+f_{-1}^{k-1}\right)-\left(\frac{572}{8192}\right)\left(f_{-1}^{k}+f_{-1}^{k-1}\right)+\left(\frac{1155}{4096}+15 \mu\right)\left(f_{-1}^{k}+f_{-1}^{k}\right)- \\
& \left(\frac{1155}{4096}+15 \mu\right)\left(f_{0}^{k}\right)+\left(\frac{572}{8192}\right)\left(f_{1}^{k}\right)-\left(\frac{63}{8192}\right)\left(f_{1}^{k}+f_{1}^{k}\right),
\end{aligned}
$$

Let $W_{k}=f_{0}^{k}-f_{-1}^{k}$ and $U_{k}=f_{-1}^{k}+f_{-1}^{k-1}$

$$
\begin{aligned}
f_{1}^{k+1}-f_{2}^{k+1}= & \left(\frac{1155}{4096}+15 \mu\right)\left(-W_{k}\right)-\left(\frac{572}{8192}\right)\left(\mathrm{U}_{k}\right)+\left(\frac{63}{8192}\right)\left(\mathrm{U}_{k-1}\right)-\left(\frac{509}{8192}\right) f_{-1}^{k-1}+ \\
& \left(\frac{509}{8192}\right) f_{0}^{k-1}+\left(\frac{1155}{4096}+15 \mu\right) f_{-1}^{k}+\left(\frac{1155}{4096}+15 \mu\right) f_{0}^{k}-\left(\frac{1155}{4096}+15 \mu\right) f_{0}^{k},
\end{aligned}
$$


after some simplification, we get the characteristic equation

$$
\begin{aligned}
& \frac{509}{8192} W_{k-1}-2\left(\frac{1155}{4096}+15 \mu\right) \mathrm{W}_{k}+\frac{63}{8192} V_{k-1}+\frac{572}{8192} V_{k}=0, \\
& \Rightarrow \frac{509}{8192}\left(\mathrm{~W}_{k-1}\right)-\left(\frac{1155}{2048}+30 \mu\right) \mathrm{W}_{k}=0 .
\end{aligned}
$$

Let $U_{k}=f_{1}^{k}-f_{-1}^{k}, W_{k}=f_{0}^{k}-f_{-1}^{k}, V_{k}=f_{1}^{k}-f_{0}^{k}, V_{k}+W_{k}=f_{1}^{k}-f_{-1}^{k}, V_{k}+W_{k}=U_{k}$

We can also write as $U_{k}=V_{k}+W_{k}, U_{k+1}=f_{1}^{k+1}-f_{-1}^{k+1}$, so

$$
\begin{gathered}
U_{k+1}=\mu f_{-3}^{k+1}+\left(\frac{77}{8192}-6 \mu\right)\left[f_{-2}^{k+1}-f_{-3}^{k+1}\right]+\left(\frac{693}{8192}-15 \mu\right)\left[f_{-2}^{k+1}-f_{-1}^{k+1}\right]+\left(\frac{3465}{4096}-\right. \\
20 \mu)\left[f_{0}^{k+1}-f_{-1}^{k+1}\right]+\left(\frac{1155}{4096}+15 \mu\right)\left[f_{-1}^{k+1}-f_{0}^{k+1}\right]+\left(\frac{495}{8192}+6 \mu\right) f_{1}^{k+1}-\mu f_{-4}^{k+1}, \\
U_{k+2}-\left(\frac{3665}{4096}-20 \mu\right) U_{k+1}=\mu V_{k}+\left(-\frac{4048}{8192}+34 \mu\right) V_{k+1}, \quad U_{k}=V_{k}+W_{k}, U_{k+1}=f_{1}^{k+1}-f_{-1}^{k+1} \\
r^{2}-\left(\frac{3665}{4096}-20 \mu\right) r=0 \Rightarrow r=\left(\frac{3665}{4096}-20 \mu\right), \\
U_{k}=c_{1}(-20 \mu)^{k}+c_{2}\left(\frac{3665}{4096}\right)^{k} .
\end{gathered}
$$

Since $U_{k+1}=f_{1}^{k+1}-f_{-1}^{k+1}$, put k=0,1 in (6) we get

$$
U_{0}=c_{1}-1+c_{2}, U_{1}=c_{1}(-20 \mu)+c_{2}\left(\frac{3665}{4096}\right)
$$

also

$$
f_{1}^{0}-f_{-1}^{0}=c_{1}+c_{2}, \quad f_{1}^{1}-f_{-1}^{1}=-20 \mu c_{1}+c_{2}\left(\frac{3665}{4096}\right)
$$

Similarly for $V_{k+2}=f_{1}^{k+2}-f_{0}^{k+2}$ we have

$$
V_{k+2}-\left(\frac{63}{8192}+\mu\right) V_{k+1}-\left(\frac{63}{8192}+\mu\right) V_{k}=0
$$

we can also write as

$$
p^{2}-\left(\frac{63}{8192}+\mu\right) p-\left(\frac{63}{8192}+\mu\right)=0
$$

Its solution is given by

$$
\begin{aligned}
& p_{1}^{\prime}=\frac{\left(\frac{63}{8192}+\mu\right)+\sqrt{\left(\frac{63}{8192}+\mu\right)^{2}+4\left(\frac{63}{8192}+\mu\right)}}{2}, \\
& p_{2}^{\prime}=\frac{\left(\frac{63}{8192}+\mu\right)-\sqrt{\left(\frac{63}{8192}+\mu\right)^{2}+4\left(\frac{63}{8192}+\mu\right)}}{2}
\end{aligned}
$$


Now we are going to establish different cases for the fractal range of (1), we have three cases in this regard

Case 1: $\quad-\frac{3360}{524288}<\mu<-\frac{3665}{81920}$

Case 2: $\quad-\frac{3665}{81920}<\mu<\frac{13024}{524288}$

Case 3: ${ }^{\mu=} \frac{-3665}{81920}$

Here we will only discuss Case 1 and others are similar.

Case 1: When $-\frac{3360}{524288}<\mu<-\frac{3665}{81920}, 7$-point binary approximating scheme gives a fractal curve.

Proof: By induction, after $k$ subdivision between $f_{0}^{0}$ and $f_{1}^{0}$, we can write as;

$$
E_{j}^{k}=f_{j}^{k}-f_{j-1}^{k}=\alpha_{1 j} f_{1}^{k}+\alpha_{2 j} f_{2}^{k}+\alpha_{3 j}(2 \omega)^{k}+\alpha_{4 j}\left(\frac{1}{2}\right)^{k}, \quad j=1,2,3,4, \ldots . ., 2^{k}
$$

where $\alpha_{i j} \neq 0, i=1,2,3,4$ we can prove that

$$
\frac{3665}{4096}<p_{2}<1,\left|p_{2}\right|>\left|p_{1}\right|,\left|p_{2}\right|>(-20 \mu)
$$

length of a vector $\mathrm{v}$ is $|v|$ and $\left|E_{j o}^{k}\right|=\min _{j=1,2,3 \ldots 2^{k}}$, we have

$$
\begin{aligned}
& \sum_{j=1}^{2^{k}}\left|E_{j}^{k}\right| \geq 2^{k}\left|E_{j o}^{k}\right|=2^{k}\left|\alpha_{1 j o} f_{1}^{k}+\alpha_{2 j o} f_{2}^{k}+\alpha_{3 j o}\left(-20 \mu^{k}\right)+\alpha_{4 j o}\left(\frac{3665^{k}}{4096}\right)\right| \\
& =\left(2 p_{2}\right)^{k}\left|\alpha_{1 j o}\left(\frac{p_{1}}{p_{2}}\right)^{k}+\alpha_{2 j o}+\alpha_{3 j o}\left(\frac{-20 \mu}{f_{2}}\right)^{k}+\alpha_{4 j o}\left(\frac{3665}{4096 p_{2}}\right)^{k}\right| \rightarrow \infty
\end{aligned}
$$

as $(k \rightarrow \infty)$.

The sum of the length of all small edges between $f_{0}^{0}$ and $f_{1}^{0}$ after $k$ iteration grows without bound when $\mathrm{k}$ tends to infinity. So when $\frac{-3360}{524288}<\mu<\frac{-3665}{81920}$, the limit curve of 7-point scheme gives fractals.

\section{Numerical Examples of 7-Point Binary Approximating Subdivision Scheme}

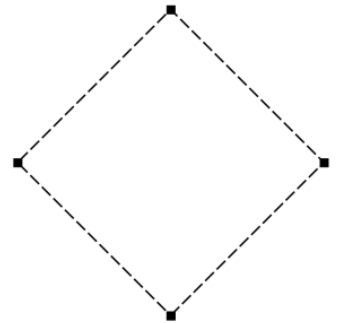

(a)

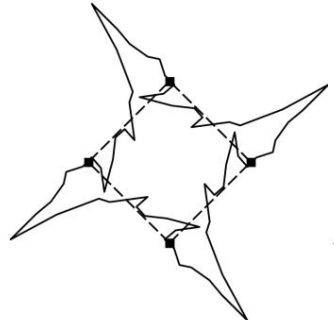

(b)

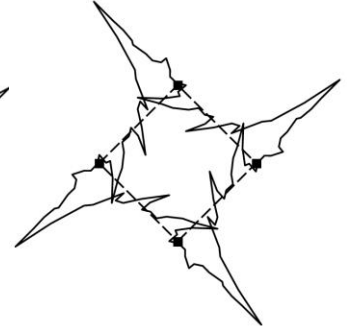

(c)

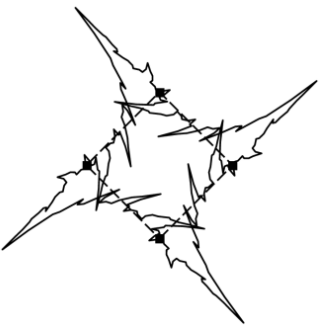

(d)

Fig. 1. (a) is the initial rhombus where solid boxes show the initial control points. (b)-(d) show the fourth, fifth and sixth refinement level at the parametric value $\mu=-0.01$ of 7-point binary approximating scheme. 


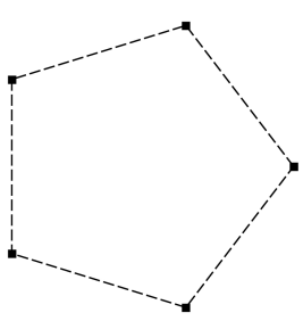

(a)

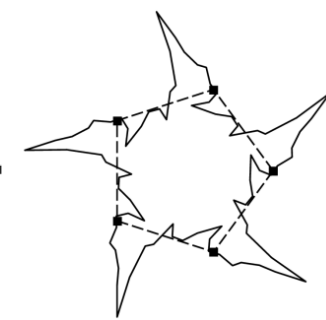

(b)

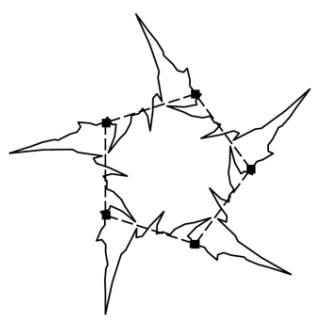

(c)

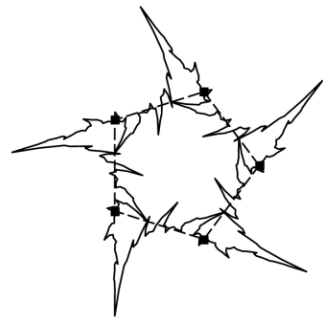

(d)

Fig. 2. (a) is the initial control pentagon where solid boxes show the initial control points. (b)-(d) show the fourth, fifth and sixth refinement level at the parametric value $\mu=-0.03$ of 7-point binary approximating scheme.

Fig. 1 (a) shows the initial rhombus and Fig. 1 (b)-(d) show the fractal generation at fourth, fifth and sixth iteration. Similarly another initial closed polygon is shown in the Fig. 2 (a) and Fig. 2 (b)-(d) show the fractal generation at fourth, fifth and sixth iteration. Fig. 1-2, we pick the value of parameter $\mu$ from the fractal range discuss in the Cases 1-3 of 7-point binary approximating scheme.

\section{Conclusion}

We calculate the fractal properties of 7-point binary approximating subdivision scheme. Fractal approach provide us the maximum deviation of limit curve instead of smoothness of the limit curve. As the scheme is parametric, so by using different values of parameter we can generate different fractal curves according to our own choice. In future, we will extend this work to regular and arbitrary topology.

\section{Acknowledgement}

This work is supported by NRPU Project No. 3183 and the SRGP Project No. 1741 of Higher Education Commission (HEC) Pakistan.

\section{References}

[1] Rham, G., (1947). Un peude Mathematiques a proposed' une courbe plane. Revwe de Mathematiques Elementry II, Oevred Completes, 2, 678-689.

[2] Dyn, N., Levin, D., \& Gregory, J. A., (1987). A 4-point interpolatory subdivision scheme for curve design. Computer Aided Geometric Design, 4(4), 257-268.

[3] Chaikin, G. M., (1974). An algorithm for high-speed curve generation. Computer Graphics and Image Processing, 3(4), 346-349.

[4] Dubuc, S., (1986). Interpolation through an iterative scheme. Journal of Mathematical Analysis and Applications, 114(1), 185-204.

[5] Boor, C., (1987). Cutting corners always works. Computer Aided Geometric Design, 4, 125-131.

[6] Weissman, A., (1990). A 6-Point Interpolatory Subdivision Scheme for Curve Design. M. Sc Thesis, Tel-Aviv University, 189-199.

[7] Romani, L., (2009). From approximating subdivision schemes for exponential splines to high-performance interpolating algorithms. Journal of Computational and Applied Mathematics, 224(1), 383-396.

[8] Zheng, H., Ye, Z., Lei, Y., \& Liu, X., (2007). Fractal properties of interpolatory subdivision schemes and their approximations in fractal generation. Chaos, Solitons and Fractals, 32, 113-123.

[9] Wang, J., Zheng, H., Xu, F., \& Liu, D., (2011). Fractal properties of the generalized Chaikin corner-cutting subdivision scheme. Computers and Mathematics with Applications, 61, 2197-2200. 
[10] Siddiqi, S. S., Siddiqui, S., \& Ahmad, N., (2014). Fractal generation using ternary 5-point interpolatory subdivision scheme. Applied Mathematics and Computation, 234, 402-411.

[11] Mustafa, G., Bari, M., Jamil, S., (2016). Engineering images designed by fractal subdivision scheme. SpringerPlus, 5(1), 1-10.

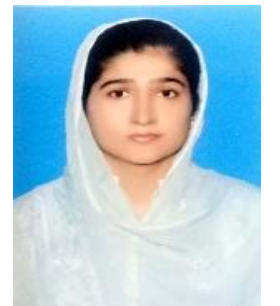

Mehwish Bari was born on $10^{\text {th }}$ January, 1984, Bahawalpur, Pakistan. After getting the basic education, she got the MSc (Mathematics) from The Islamia University of Bahawalpur, Pakistan in 2005. She getting the Scholarship for M.Phil leading to the Ph.D. in 2006 from Higher Education Commission Pakistan, complete M.Phil (mathematics) in 2009 and Ph.D. (mathematics) [computer aided geometric design] in 2016 from The Islamia University of Bahawalpur, Pakistan.

Dr. Bari worked as research associate in the Department of Mathematics, the Islamia University of Bahawalpur, Pakistan from 4th March, 2015 to $3^{\text {rd }}$ March, 2018.

Dr. Bari worked as visiting lecturer, Department of Physics and Computer Science, the Islamia University of Bahawalpur, Pakistan from February 2016 to June 2017.

Dr. Bari Currently working as chairperson, Department of Mathematics, National College of Business Administration and Economics, Sub-Campus, Bahawalpur. Dr. Bari is a member of Organizing Committee, International Pure Mathematics Conference.

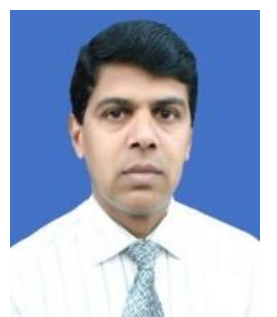

Ghulam Mustafa was born on $5^{\text {th }}$ January, 1973, Bahawalpur, Pakistan. After getting the basic education, He got the MSc and M.Phil. (mathematics) degrees from Bahauddin Zakariya University Multan, Pakistan in 1993 and 1996 respectively. He received a Ph.D. (2004) in mathematics from the University of Science and Technology of China, P. R. China. He did the post doctorate from Durham University UK sponsored by Association of Commonwealth Universities UK 2011. He was visiting fellow at University of Science and Technology of China from 2013-2014 sponsored by Chinese Academy of Sciences.

Currently, he is full professor of mathematics and chairman Department of Mathematics at The Islamia University of Bahawalpur, Pakistan. His research interests include geometric modelling, applied approximation theory.

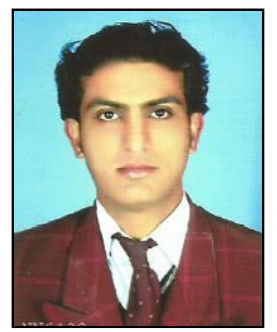

Abid Hussain was born in 09/11/1985, Muzaffargarh, Pakistan. After getting the basic education, he got the MSc mathematics degree in 2009 from the Islamia University of Bahawalpur, Pakistan. He did M. Phil mathematics in 2016 from NCBA\&E Sub-Campus Bahawalpur, Pakistan.

Mr. Hussain is working as elementary school teacher, Punjnad colony school, district Muzaffargarh, tehsil Alipur, Pakistan. 